\title{
ANÁLISE DA EDUCAÇÃO DE CLIENTES/PACIENTES NA LITERATURA BRASILEIRA DE ENFERMAGEM
}

\author{
Adriana Meneghini Melles* \\ Márcia Maria Fontão Zago**
}

MELLES, A.M.; ZAGO, M.M.F. Análise da educação de clientes/pacientes na literatura brasileira de enfermagem. Rev.latino-am.enfermagem, Ribeirão Preto, v. 7, n. 5, p. 85-94, dezembro 1999.

Esse estudo tem o objetivo de identificar os aspectos conceituais e operacionais da educação de clientes/pacientes na literatura brasileira de enfermagem. Foram analisados 60 trabalhos publicados no periodo 1980 a 1995 . Os dados foram codificados e categorizados. A nível conceitual, a atividade educativa é caracterizada como uma ação instrumental de informação e de proteção para o paciente. A atividade é rotulada como orientação e segue pressupostos teóricos contraditórios, focalizando a doença. Operacionalmente, a atividade é desenvolvida como ação informativa e não de conscientização, e a avaliação é inadequada.

UNITERMOS: educação de pacientes, enfermagem

\section{INTRODUÇÃO}

A atividade educativa do enfermeiro com o cliente/paciente é considerada antiga e teve origem na Enfermagem Moderna, com Florence Nightingale, no século XIX ${ }^{1}$.

Com as transformações sócio-culturais ocorridas nas últimas décadas, a atividade educativa do enfermeiro tem sido destacada como fundamental para a promoção e manutenção da saúde. Na situação de doença, ela é estratégica para a obtenção da participação do cliente/ paciente no tratamento e reabilitação ${ }^{12}$.

$\mathrm{Na}$ literatura de enfermagem, essa atividade é rotulada como educação de cliente/paciente ${ }^{2}$.

$\mathrm{Na}$ atualidade, há diferentes modelos teóricometodológicos que podem ser aplicados à educação de pacientes. Entretanto, pouco sabemos como os enfermeiros brasileiros estão fundamentando e realizando a atividade.

A proposta desse estudo é desvelar os pontos obscuros, as lacunas, as congruências e incongruências sobre a atividade. Sistematizar o conhecimento sobre a atividade, pela literatura, tem importância teórica (para compreensão da realidade) e prática (executá-la com eficiência) para a profissão. Assim, o objetivo do estudo é:
- identificar os aspectos conceituais e operacionais da atividade educativa do enfermeiro com o paciente/ cliente, na literatura de enfermagem, no período de 1980 a 1995.

As definições conceituais fornecem as prioridades abstratas dos termos (filosofia, abordagem teórica). As definições operacionais apontam os indicadores concretos, específicos (fatos, qualidades, ações) ${ }^{8}$.

\section{METODOLOGIA}

A dissertação argumentativa, a que se propõe este estudo, constitui-se num estudo bibliográfico em que os pesquisadores apresentam uma exposição compreensiva do tema, interpretando as idéias expostas e a posição pessoal dos autores, na literatura, através da apresentação de razões e evidências, segundo os princípios da argumentação ${ }^{8}$.

Para o alcance do objetivo proposto, o estudo percorreu as seguintes etapas:

1 - Levantamento bibliográfico através da fonte bibliográfica "Lilacs" (que reúne a literatura latinoamericana em ciências da saúde), partindo dos unitermos: educação, ensino e orientação de paciente/cliente, e

\footnotetext{
* Aluna do Curso de Graduação em Enfermagem. Escola de Enfermagem de Ribeirão Preto da Universidade de São Paulo. Bolsista PIBIC/USP/CNPq

** Enfermeira. Docente do Departamento de Enfermagem Geral e Especializada da Escola de Enfermagem de Ribeirão Preto da Universidade de São Paulo. Orientadora do Trabalho
} 
atividade educativa. O período abrangido foi de 1980 a 1995.

2 - Localização das publicações: através de empréstimo, consulta e reprodução de cópias.

3 - Análise e síntese do material:

3.1 - leitura informativa ou exploratória: consistiu na leitura do material;

3.2 - leitura seletiva: consistiu na discriminação do material quanto a sua pertinência ao estudo ou não;

3.3 - leitura crítica ou reflexiva: consistiu na busca pelas definições conceituais e operacionais da atividade educativa apresentadas pelos autores dos textos, através da apreensão, análise, compreensão e julgamento das definições;

3.4 - leitura interpretativa: consistiu no julgamento da pertinência ou não das afirmações e dados apresentados; 3.5 - preenchimento das fichas bibliográficas: consistiu no translado das informações e definições para uma ficha de síntese das leituras (Anexo 1);

3.6 - síntese integradora: consistiu na construção de um esquema integrador dos resultados.

Após a leitura crítica, os dados foram digitados no computador, através de um programa redator e em seguida foram transferidos para o programa THE ETHNOGRAPH ${ }^{2}$. Neste programa os dados foram codificados em: princípio, finalidade, nome, tipo de paciente, autocuidado, ação, estratégia. Esses códigos foram integrados em duas categorias: definições conceituais, definições operacionais. Essas categorias forneceram a síntese integradora dos dados, ou seja, os resultados.

\section{RESULTADOS E DISCUSSÃO}

\section{A - Descrição geral}

No levantamento realizado, foram identificados 127 trabalhos publicados, no período de 1980 a 1995 , nos seguintes periódicos: Revista Brasileira de Enfermagem, Revista da Escola de Enfermagem de São Paulo, Acta Paulista, Revista Gaúcha de Enfermagem. Essa identificação partiu da presença das palavras educação, ensino, orientação e atividade educativa entre os unitermos, no título ou corpo do trabalho. Estes periódicos foram selecionados por serem considerados os de maior periodicidade e inserção na profissão. Foram excluídos os trabalhos de autoria de alunos de graduação.

Após sucessivas leituras de todos os trabalhos, selecionamos os que enfocavam o tema com maior abrangência, nos quais poderíamos identificar as definições conceituais e/ou operacionais. Dessa forma, a seleção final resultou em 60 publicações (Anexo 2).
O primeiro aspecto a ser considerado refere-se ao número de publicações durante intervalos de anos (Figura 1). Percebemos que não há diferença significativa na freqüência de publicações relacionadas aos períodos de anos. Pelo contrário, as publicações sobre o tema são constantemente apresentadas.

Os trabalhos selecionados foram classificados em: artigos (estudos teóricos, análises críticas, ensaios) e pesquisas (relatos de experiência e estudos metodológicos). Classificamos 27 artigos e 33 pesquisas. Chama-nos a atenção o número de relatos de experiência que é de 22, tendo maior freqüência no período de 1980 a 1985.

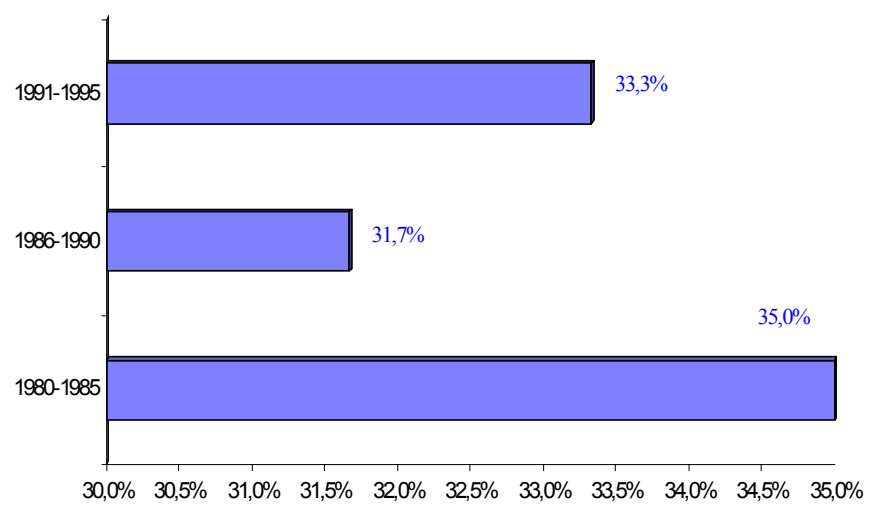

Figura 1 - Localização temporal das publicações

Pelos números apresentados podemos afirmar que tem havido investimento dos enfermeiros em descrever e divulgar suas experiências de educação de paciente nos periódicos.

Quanto a categoria profissional dos autores, os resultados mostram que dos 54 autores, 26 atuam na área hospitalar e 28 na área de saúde coletiva, ou comunitária. Entre os autores, 33 são profissionais da área da docência, ou seja, os docentes de enfermagem privilegiam a pesquisa em educação do paciente (alguns autores publicaram mais de um estudo).

Nos trabalhos selecionados, o posicionamento dos autores em relação a função do enfermeiro como educador é bastante destacado, como observamos nas afirmações:

\footnotetext{
"A ação educativa deve ser adotada como uma postura profissional, um compromisso com a realidade de saúde da população brasileira."(21 A)

"A educação em saúde favorece experiências muito importantes na formação do futuro profissional para quem a tarefa de ensinar é uma das funções primordiais." $(6 P)$
} 
"A enfermeira precisa desempenhar seu papel de orientadora e educadora." (7 A) a formação e no próprio cotidiano profissional, mostrando que esse aspecto tem, também, um cunho cultural.
Portanto, o enfermeiro brasileiro considera-se educador do cliente/paciente por excelência, corroborando os resultados de pesquisadores internacionais $^{2,6,7,11}$. A atividade educativa do enfermeiro com o cliente/paciente é valorizada na literatura, durante

\section{B - Definições conceituais}

A nível conceitual, a atividade educativa tem recebido diferentes denominações e, em alguns trabalhos, dois ou três nomes são utilizados como sinônimos:

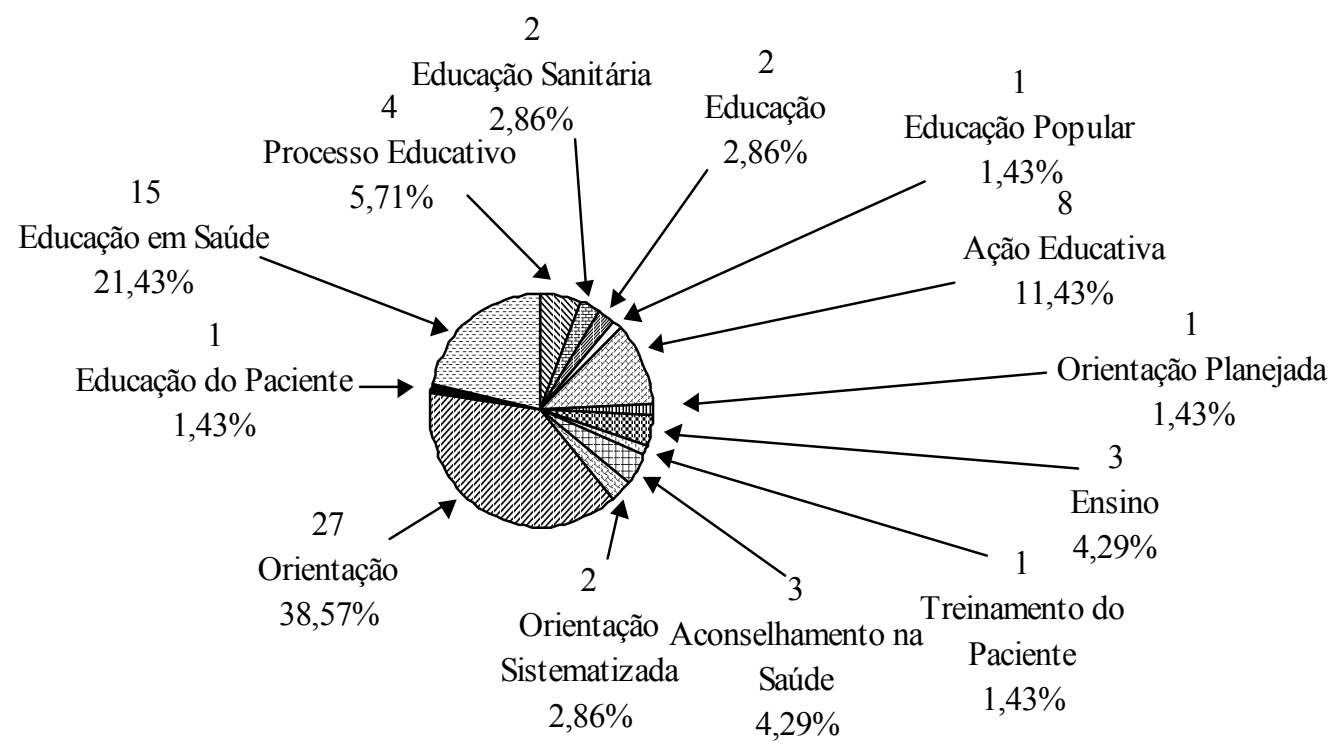

Figura 2 - Freqüência dos rótulos dados à atividade educativa

Entre as denominações utilizadas, o rótulo "orientação", como mostra a Figura 2, encontra-se citado em maior freqüência $(38,5 \%)$. O termo orientação é o que identifica a atividade, não apenas na literatura mas, também, na linguagem coloquial profissional. ZAGO ${ }^{12}$ descreve o processo de orientar como um ritual. Enquanto ritual, o seu significado simbólico determina características, valores, conhecimentos, crenças e padrões culturais que foram relacionadas com as funções sociais, psicológicas e protetoras do ritual, na visão dos enfermeiros. Segundo a autora, a desvantagem da prática ritualística da orientação, está na negação do paciente como agente ativo do processo, com suas singularidades, com suas necessidades específicas de aprendizagem, tendendo a ser excluído do processo ou pouco considerado. Assim, esse paradigma não se relaciona com o processo de ensinar e aprender que pressupõe os conceitos teóricos da educação de paciente.

Segundo BARTLETT ${ }^{2}$, a educação de pacientes é uma experiência planejada, usando uma combinação de métodos como ensino, aconselhamento e técnicas de modificação de comportamento, que influenciam o conhecimento do paciente e, conseqüentemente, o seu comportamento de saúde. A mesma autora diferencia essa atividade a de aconselhamento. $\mathrm{O}$ aconselhamento é um processo individualizado, envolvendo direcionamento e solução de problemas colaborativos para ajudar o paciente a melhor lidar com o problema de saúde. Para RANKIN $\&$ STALLINGS $^{6}$, a diferenciação entre educação e ensino de pacientes repousa no conteúdo, sendo que no primeiro o conteúdo é mais amplo, focalizando a saúde. $\mathrm{Na}$ segunda situação, o conteúdo é mais específico, delimitado ao problema de saúde apresentado pelo paciente.

A terminologia não concisa entre os pesquisadores tal como orientação, educação, ensino, podem levar à uma inadequada compreensão teórica e prática.

Ao descreverem a finalidade do processo educativo os enfermeiros apresentam pressupostos do Modelo Preventivo de Saúde (fundamentado no Modelo Médico ou Tradicional) associado ao Modelo Pedagógico Humanístico (fundamentado em Paulo Freire), implícitos nas afirmações abaixo ${ }^{4,6,9,10,11}$.

"Educação em saúde é a mudança de atitude baseada na conscientização da sua realidade." $(8 \mathrm{~A})$

"Quanto maior o conhecimento da pessoa sobre sua saúde, mais condições apresenta para questionar a natureza de seu problema, 
o que facilita a adaptação ao seu estado de saúde, aumentando sua cooperação com o regime terapêutico e assim, facilitando sua independência" (10 A)

"Quando são empreendidas ações educativas junto aos clientes, estas freqüentemente visam atingir os objetivos do profissional que, baseado em suas necessidades ou sobre o que acredita ser importante para o cliente, familia ou comunidade, traça o seu programa de orientação. As ações educativas visam o autocuidado que desenvolve o sentimento de independência, de autocontrole e autodeterminação".(15 P)

"Pesquisas realizadas comprovaram que a participação da enfermeira junto a programas de orientação de hipertensos contribuem para o aumento do índice de aderência ao tratamento".(22 P)

Relacionando-se as finalidades e as conseqüências das atividades descritas pelos autores, delimitamos os três atributos ou qualidades dados à educação de pacientes:

- ação instrumental: o processo é concebido como um instrumento que produzirá e/ou influenciará mudanças de comportamento e de atitude no paciente:

"A orientação pré - operatória influencia as principais alterações apresentadas pelos pacientes em pós-operatório imediato e proporciona melhor recuperação." (1 P)

“ A orientação a nivel ambulatorial pode reduzir o número de internações devido ao melhor conhecimento, pelos pacientes, dos efeitos tóxicos produzidos pelos quimioterápicos."(3 P)

"Educação em saúde é a mudança de atitude baseada na conscientização da sua realidade." $(8 \mathrm{~A})$

- ação informativa: o fornecimento da informação é um elemento importante na educação de pacientes e é ela que levará à mudanças no comportamento do paciente:

"Educação em saúde é incentivar a apresentação voluntária de doentes, eliminar falsos conceitos, informar quanto a sintomatologia, estimular a assiduidade do doente, dar conhecimento dos locais de tratamento." (8 A)

- ação protetora: através da educação o paciente pode ter sua condição física e emocional equilibrada:

"Da mesma maneira que a cirurgia, os exames diagnósticos causam uma certa apreensão no paciente, a qual pode ser minimizada com a orientação realizada pela enfermeira." $(33 P)$

As afirmações acima realçam a contradição conceitual ou filosófica do processo. Os dados denotam que os enfermeiros tem uma visão individualística da doença ${ }^{9,11}$, ou seja, a doença tem como causa aspectos internos ao indivíduo, desconsiderando os aspectos sociais e ambientais. Outra contradição refere-se a utilização de termos como conscientização, autocuidado, transformação e aderência, adesão e reforço, que são termos conceituais de visões filosóficas distintas. Exemplificando, a adesão ao tratamento implica numa atitude passiva de aquiescência e não de conscientização $^{11}$.

Há uma diversidade de pressupostos filosóficos para a educação de pacientes. TONES et al. ${ }^{11}$, consideram que ao longo dos anos houve um predomínio dos defensores do Modelo Preventivo de Saúde e, em segundo lugar, dos seguidores da Visão Educacional Humanística. $\mathrm{Na}$ literatura brasileira de enfermagem não é diferente, as duas concepções são relacionadas, porém, são contraditórias: a) a concepção centrada no modelo médico (no qual a aceitação ou adesão ao tratamento é a palavra chave e cuja linguagem tem os significados de aceitação, adesão, planejamento para os pacientes, mudança de comportamento, paciente passivo, dependência, necessidades determinadas pelos profissionais); b) e a centrada no paciente (a palavra chave é autonomia e a linguagem tem os significados de independência, participação do paciente no planejamento, paciente ativo, conscientização e emancipação).

\section{C - Definições operacionais}

As características operacionais só puderam ser analisadas em alguns dos relatos de experiência e pesquisas.

A atividade educativa desenvolvida pelos enfermeiros, mostra-se multidimensional no sentido que abrange diferentes grupos de pacientes: diabéticos (7), cirúrgicos (5), hipertensos (5), gestantes (4), enfartados (4) e outros. Frente a esse aspecto, os pacientes diabéticos são os que estão recebendo maior atenção quanto à sua educação em saúde, pelo enfermeiro. Esse resultado 
corresponde aos de outros estudos da literatura internacional $^{3,4,9}$, que considera que a educação em saúde com o paciente portador de diabetes tem sido pesquisado em maior profundidade. Segundo RANKIN \& STALLINGS $^{6}$ é nessa área que a enfermagem tem tido reconhecimento por estar expandindo um modelo de Educação em Saúde. Por outro lado, esses autores destacam que os pacientes com doenças crônicas, de modo geral, têm recebido maior atenção educativa pelos enfermeiros.

Outra característica é que os estudos focalizam, na sua maioria, o ser doente (paciente). Apenas três estudos eram direcionados para os clientes, indivíduos sem problema de saúde real.

Enquanto uma ação instrumental, a operacionalização da educação de clientes/ pacientes é identificada, pelos autores, de diferentes modos:

“... é uma atividade sistemática e dirigida a pacientes e seus contatos..."(4 P)

"A educação como estratégia para reinserção do indivíduo deficiente..." (7 A)

"O trabalho educativo do paciente e da família é um processo contínuo ..." (32 P)

"A orientação planejada de enfermagem para a alta hospitalar é mais uma fase no processo de sistematização da assistência de enfermagem..." (35 A)

Por processo, estratégia ou sistematização, entendemos que a atividade é flexível, tem uma continuidade e busca um objetivo definido, coerente com a definição de processo educativo e de ensino/ aprendizagem $^{1,2}$.

Quanto ao objetivo dos programas desenvolvidos, os mais citados foram: desenvolvimento do autocuidado, transformação da realidade e conscientização para a saúde (abordagem humanística). O autocuidado, como objetivo, foi citado em 18 (30\%) dos trabalhos, a conscientização 4 vezes $(6,6 \%)$ e a transformação da realidade uma vez $(1,6 \%)$, entre os 60 trabalhos. Esses objetivos ressaltam as abordagens conceituais utilizadas pelos autores, referidas anteriormente nesse estudo.

Através dos conteúdos relacionados nos estudos podemos caracterizar, operacionalmente, a ação informativa do processo. O conteúdo dos programas educativos apresentados foram classificados em dois tipos: de informações processuais e sensoriais ${ }^{11}$. As informações processuais descrevem a natureza dos procedimentos terapêuticos, quando, onde e por quem será realizado e os riscos. As informações sensoriais descrevem as sensações que o paciente pode esperar. $\mathrm{O}$ conteúdo abrangido é essencialmente da área temática da doença e não da visão de saúde, como pode-se perceber nas afirmações abaixo:

"A enfermeira orienta quanto à administração e possíveis efeitos tóxicos; a rotina de funcionamento e quanto ao tratamento domiciliar..."(3 P)

“A orientação foi padronizada quanto ao seguinte conteúdo: finalidade do exame, duração do exame, reações e sensações sentidas, descrição sumária da técnica empregada, e como poderá colaborar com o exame." (5 P)

“ ...orientação quanto a alimentação, higiene, hábitos e recreação." (13 A)

"Na orientação do paciente para alta, as orientações são dadas pela enfermeira, com a presença de um familiar é é demonstrado o preparo da ciclosporina (material necessário), enfocado questões de higiene domiciliar. São reforçadas as orientações sobre o plano a ser seguido e a importância do retorno para controle médico." (26 P)

“... o fornecimento de informações, esclarecer dúvidas, atenção ao aspecto emocional, demonstrações dos procedimentos." (42 A)

Novamente ressaltamos que o conteúdo dos programas não abordam os aspectos sócio/ambientais da doença e nem conteúdos que possam realmente propiciar o desenvolvimento do autocuidado e da conscientização. Assim, operacionalmente, os enfermeiros não implementam os conceitos apresentados nas definições conceituais anteriormente mencionadas.

Quanto aos recursos utilizados, foram citados os seguintes: demonstrações, palestras, álbuns seriados, cartazes, manual de informação, diálogo e demonstração com devolução. Uma das grandes falhas dos trabalhos analisados foi a não explicitação da justificativa para a utilização desses recursos, em relação aos grupos de pacientes a que foram aplicados, como também não há uma avaliação do emprego desses recursos. Chama-nos a atenção que apenas dois relatos de experiência citaram a utilização de folhetos ou manuais de informação, mas sem apresentar a forma como foram elaborados. A informação escrita pode ajudar o conhecimento dos pacientes quanto ao seu diagnóstico, reforçando a 
informação oral já dada; pode servir como lembrança para referência futura, servindo como estímulo para questões e prevenindo má compreensão sobre o diagnóstico e tratamento ${ }^{5}$. Entretanto, a produção de folhetos informativos requer uma série de cuidados quanto à adequação de conteúdo, linguagem e lay-out ${ }^{1,5,6}$.

$\mathrm{Na}$ avaliação dos programas e das experiências implementadas, há inadequações em todos os aspectos. A avaliação não é uma mera atividade técnica, ela incorpora aspectos filosóficos e requer compreensão do propósito da pesquisa e do significado do sucesso. Diferentes modelos metodológicos tem seus defensores e críticas mas, para a maioria dos pesquisadores, as decisões sobre os modelos de avaliação são determinados por dois fatores principais: as questões a serem respondidas e os problemas situacionais do processo $^{3,4,6,11}$.

A avaliação somativa está preocupada com a questão do sucesso do programa educativo realizado. Neste parâmetro, os métodos adequados são o experimental (grupo controle) e o survey; ambos apresentam princípios metodológicos específicos ${ }^{4,11}$.

Para a avaliação formativa a preocupação é com os detalhes da implementação dos programas. Neste caso o método etnográfico é considerado o mais adequado ${ }^{4,11}$.

Os métodos citados acima têm limitações e atualmente é reconhecido a necessidade de associar-se metodologias para se avaliar o sucesso do processo de educação de pacientes, com a descrição dos seus indicadores ${ }^{4,11}$.

Entre os estudos selecionados, apenas dois apresentam uma abordagem metodológica de avaliação adequada (método experimental). Nos outros estudos, os autores avaliaram informalmente o processo como de sucesso, através das descrições de expressão de satisfação pelo paciente e da própria enfermeira com a experiência. Mesmo aqueles que desenvolveram questionários e entrevistas de avaliação, estes não foram submetidos a qualquer tipo de análise estatística ou qualitativa. Conforme afirmado pelos autores, houve apenas a preocupação em avaliar a aplicação imediata das aprendizagens desenvolvidas no final do programa e não a longo prazo.

Frente a esses resultados, consideramos que os resultados apresentados pela maioria dos autores não são parâmetros objetivos com os quais se pode afirmar que os programas realizados tiveram sucesso e que conseguiram atingir os objetivos e pressupostos conceituais inicialmente apresentados.

\section{CONSIDERAÇÕES FINAIS}

O presente estudo teve como propósito dar continuidade aos nossos estudos sobre Educação de Pacientes. Buscamos, através da literatura nacional, realizarmos uma aproximação geral do tema, sem ter a pretensão de esgotar o assunto.

Apresentamos um panorama dos artigos e pesquisas sobre o tema, desvelando seus aspectos característicos, que podem levar à reflexões teóricas e práticas.

Com a seleção de 60 estudos, envolvendo artigos e pesquisas, buscamos identificar os aspectos conceituais e operacionais que fundamentaram os trabalhos sobre o tema.

Os resultados obtidos levam-nos a considerar que:

- A educação de paciente é uma ação instrumental e de proteção, do enfermeiro para com o paciente, em que o fornecimento de informação (orientação) é sua essência. Entretanto, educar implica no desenvolvimento de outras hierarquias de aprendizagem (habilidades psicomotoras e atitudes), e todas elas associadas é que poderão constituir-se em subsídios para as alterações de comportamentos e atitudes de saúde.

- Pelos conteúdos desenvolvidos nos programas educativos, o enfermeiro abrange apenas temas relacionados à doença e ao tratamento. Esses conteúdos são fragmentados e não há abordagem aos aspectos sociais e ambientais da doença. A informação, por si só, não garante uma mudança de comportamento. Nesse sentido, concordamos com BARTLETT ${ }^{1}$ de que a educação do paciente deveria ser dirigida para o que o paciente precisa fazer para ter saúde e não apenas pelo que ele deve saber. Conseqüentemente, o processo tem como foco o indivíduo e não o ser social.

- Operacionalmente, o desenvolvimento de programas educativos tem sido realizado tanto a nível hospitalar como comunitário; com contradições teóricometodológicas. Há maior número de experiências com o ser doente (paciente) em relação ao ser saudável; isto é, a enfermagem brasileira é mais atuante no ensino de pacientes do que na educação de clientes. A abordagem teórica dos programas é humanista, mas segue princípios pedagógicos comportamentalistas. A avaliação é o procedimento que menor respaldo dá para as afirmações de sucesso do programa.

Finalizando, as pesquisas e relatos de experiência, publicados nos periódicos brasileiros, falham a nível teórico e metodológico e pouco favorecem para o desenvolvimento do conhecimento sobre o processo.

Acreditamos que seja o momento dos enfermeiros repensarem sobre a sua prática educativa com o cliente/paciente. Será que não é hora de facilitarmos a educação do paciente, ou devemos continuar a orientálo? 


\section{ANALYSIS OF THE CLIENTS/PATIENTS EDUCATION IN THE BRAZILIAN NURSING} LITERATURE

This study aims to identify the conceptual and operational aspects of the clients/patients education, in the brazilian nursing literature. Sixty studies published between 1980 and 1995 were reviewed. The data were analysed and coded. At the conceptual level, the education activity is characterized as a mechanical action of information and of patients' protection. It is seen as orientation and follows contradictory conceptual frameworks focussing the illness. At the operational level, the activity is developed as an informative action and not as a consciousness one, and the evaluation is inadequate.

KEY WORDS: patient education, nursing

\section{ANÁLISIS DE LA EDUCACIÓN DE CLIENTES/PACIENTES EN LA LITERATURA BRASILEÑA DE ENFERMERÍA}

Este estudio tiene como objetivo verificar los aspectos conceptuales y operacionales de la educación del clientes/pacientes en la literatura brasileña de enfermería. Fueron analizados 60 trabajos in publicaciones en el periodo de 1980 a 1995 . Los datos fueron codificados y categorizados. A nivel conceptual, la actividad educativa es caracterizada como una acción instrumental de información y de protección para el paciente. La actividad es rotulada como orientación y sigue presupuestos teóricos contradictorios, focalizando la enfermedad. Operacionalmente, la actividad es desarrollada como una acción informativa y no como concientización, y la evaluación es inadecuada.

TÉRMINOS CLAVES: educación del paciente, enfermería

\begin{tabular}{ll}
\hline \multicolumn{1}{l}{$\begin{array}{l}\text { ANEXO 1 } \\
\text { FICHA BIBLIOGRÁFICA }\end{array}$} & \multicolumn{1}{c}{ - CATEGORIA PROFISSIONAL: } \\
1- IDENTIFICAÇÃO: & 3- SÍNTESE DA PUBLICAÇÃO: \\
- AUTOR: & \\
- TÍTULO: & 4- CARACTERIZAÇÃO DO CONTEÚDO: \\
- TIPO DE PUBLICAÇÃO: & - OBJETIVO DO ARTIGO OU ESTUDO: \\
- ANO: & - DEFINIÇÕES CONCEITUAIS: \\
2- CARACTERIZAÇÃO DA PUBLICAÇÃO: & - DEFINIÇÕES OPERACIONAIS: \\
- CATEGORIA PROFISSIONAL DO AUTOR: & - RESULTADOS OBTIDOS: \\
- ÁREA DE ATUAÇÃO: & - CONCLUSÕES: \\
- CO-AUTORES: & \\
- NÚMERO: & 5- OBSERVAÇÕES:
\end{tabular}

\section{ANEXO 2 \\ RELAÇÃO DOS TEXTOS ANALISADOS}

AGUILlAR, O.M.; ANGERAMI, E.L.S. Avaliação da orientação sistematizada na reabilitação da voz de paciente laringectomizado. Rev.Esc.Enfermagem USP, São Paulo, v. 17, n. 1, p. 47-60, 1983.

ANDREONI, S.; SILVA, S.H.; FERNANDES, R.A.Q. Programa de assistência domiciliar do hospital universitário da USP. Rev.Bras.Enfermagem, v. 47, n. 3, p. 295-303, jul./set. 1994.

AZEVEDO, M.A.J.; MARIA, M.L.S.S.; SOLER, L.M. A. Promovendo o auto - cuidado - treinamento e assistência de enfermagem a pacientes portadores de bexiga neurogênica. Rev.Bras.Enfermagem, Brasília, v. 43, n. 1,2,3/4, p. 52-57, jan./dez. 1990.
BARROS, A.S.; CHRISTÓFORO, F.F.M. Abordagem do enfermeiro no grupo educativo com gestantes candidatas à laqueadura. Rev.Bras.Enfermagem, Brasília, v. 36, n. 2, p. 101-106, abr.jun. 1993.

BOTTO, M.C.A. et al. Orientação para tratamento domiciliar de enema com corticóide à paciente portador de retite actníca pós-radiomoldagem. Rev.Bras.Enfermagem, Brasília, v. 34, n. 1, p. 62-65, 1981.

CAR, M.R.; PIERIN, A.M.G.; AQUINO, V.L.A. Estudo sobre a influência do processo educativo no controle da hipertensão arterial. Rev.Esc.Enfermagem USP, v. 25, n. 3, p. 259-269, dez. 1991.

CÁRNIO, A.M.; CINTRA, F.A.; TONUSSI, J.A. Orientação pré - operatória a pacientes com catarata e indicação de cirurgia ambulatorial - relato de experiência. Rev.Bras.Enfermagem, Brasília, v. 48, n. 1, p. 39-45, jan./ mar. 1995. 
CARVALHO, Z.M.de F. Orientação de enfermagem. Fator importante no ajustamento das mulheres mastectomizadas: contribuição à assistência de enfermagem. Rev.Bras.Enfermagem, Brasília, v. 37, n. 3/4, p. 157-164, jul./dez. 1984.

CEZARETTI, I.U.R. et al. Estudo preliminar do conhecimento do paciente a respeito de esôfago - gastro - duodenoscopia como base para orientação de enfermagem. Acta Paul.Enfermagem, São Paulo, v. 1, n. 2, p. 42-47, abr./ jun. 1988.

. Ostomizado: reabilitação sem fronteiras? Ponto de vista do enfermeiro. Rev.Bras.Enfermagem, Brasília, v. 48, n. 1, p. 60-65, jan./mar. 1995.

CIOSAK, S.I. et al. Estudo preliminar sobre a influência da Orientação sistematizada ao paciente que será submetido ao estudo hemodinâmico. Rev.Bras.Enfermagem, Brasília, v. 35,n. 1, p. 17-38, jan./mar. 1982.

COLLET, M.I.A. et al. Estudo sobre pacientes de hanseníase com incapacidades físicas, tratados através de técnicas simples, aplicado pelo pessoal de enfermagem do D. F. em 1979. Rev.Bras.Enfermagem, Brasília, v. 34, n. 1, p. 7899, jan./mar. 1981.

CRISTOFOLINI, L. Prevenção de incapacidades na hanseníase. Rev.Bras.Enfermagem, Brasília, v. 35, n. 3 e 4, p. 226237, julho/dez. 1982.

DYTZ, J.L.G; CRISTO, R.C. A ludoteca como espaço para uma nova abordagem de educação em saúde. Rev.Bras.Enfermagem, Brasília, v. 48, n. 2, p. 134-139, abr./jun. 1995.

ÉVORA, Y.D.M. et al. Uma proposta para a educação da gestante na assistência pré - natal, com base no enfoque sistêmico. Rev.Gaúch.Enfermagem, Porto Alegre, v. 8, n. 2, p. 261-269, jul. 1987.

Orientações ministradas à gestante durante a assistência pré - natal: atuação dos profissionais de enfermagem. Rev.Esc.Enfermagem USP, São Paulo, v. 22, n. 3, p. 339-351, dez. 1988.

FERNANDEZ, H.C. et al. O enfermeiro no Ambulatório de Oncologia Clínica. Rev.Bras.Enfermagem, Brasília, v. 34, n. 1, p. 66-70, 1981.

FONTES, M.C.C.; ALMEIDA, M.S.; CARVALHO, D.D. O trauma cirúrgico: importância da orientação pré-operatória. Rev.Bras.Enfermagem, Brasília, v. 33, n. 2, p. 194-200, abr./jun. 1980.

GIR, E.; MORIYA, T.M.; ROBAZZI, M.L.C.C. Ações educativas e doenças sexualmente transmissíveis. Rev. Gaúch.Enfermagem, Porto Alegre, v. 12, n. 1, p. 38-40, jan. 1991.

GUIMARÃES, M.L.M.V. Participação do enfermeiro no treinamento do paciente lesado raquimedular e usuário de farmacoterapia intracavernosa peniana: papaverina. Rev.Bras.Enfermagem, Brasília, v. 44, n. 2/3, p. 116-118, abril/set.1991.

IDE, C.A.C.; CNAVES, E. C. Saúde e educação enquanto prática sociais. Rev.Esc.Enfermagem USP, São Paulo, v. 24, n. 2, p. 225-236, ago. 1990.

KAMIYAMA, Y. et al. Educação para a saúde no controle de doenças transmissíveis: uma experiência de integração hospital - escola de enfermagem. Rev.Bras.Enfermagem, Brasília, v. 1, p. 74-80, 1982.
LAGANÁ, M.T.C. A educação para a saúde: o cliente como sujeito da ação. Rev.Esc.Enfermagem USP, São Paulo, v. 23, n. 1, abr. 1989.

LAGANÁ, M.T.C. et al. Auto exame de mama: identificação dos conhecimentos, atitudes, habilidades e práticas (CAPH) requeridos para elaboração de propostas educativas. Rev.Esc.Enfermagem USP, São Paulo, v. 24, n. 2, p. 281299, ago. 1990.

LINDHOLM, R.R. Cuidado do lactente no primeiro ano de vida - conhecimentos desejados por um grupo de mães. Rev.Bras.Enfermagem, Brasília, v. 37, n. 1, p. 36-43, jan./ mar. 1984.

LUCE, M. et al. O preparo para o auto - cuidado do cliente diabético e família. Rev.Esc.Enfermagem USP, São Paulo, v. 25, n. 2, p. 137-152, ago. 1991.

MARRA, C.C. et al. Orientação planejada de enfermagem na alta hospitalar. Acta Paul.Enfermagem, v. 2, n. 4, p. 123127, dez. 1989.

MARTINO, M.M.F. et al. Manual de orientação para paciente infartado. Rev.Bras.Enfermagem, Brasília, v. 47, n. 3, p. 304-313, jul./set. 1994.

MILANEZ, H.M.; ALMERÃO, E. O papel da enfermeira na orientação e administração de drogas que levam a imunossupressão em pacientes submetidos a transplante cardíaco. Rev. Gaúch.Enfermagem, Porto Alegre, v. 8, n. 2, p. 220-226, jul. 1987.

MUSSI, F.C.; GUERRERO, M.; CARAMELLI, B. Infarto agudo do miocárdio: manual de orientação para o paciente. Rev.Bras.Enfermagem, Brasília, v. 29, n. 2, p. 158-165, ago. 1995.

NEVES, T.A. et al. O papel do (a) enfermeiro (a) do trabalho na reinserção social da pessoa com deficiência. Rev.Bras.Enfermagem, Brasília, v. 35, n. 3/4, p. 192-199, julho/dez. 1982.

NUNES, D.M. et al. Aspectos da atividade assistencial da enfermagem na unidade de internação pediátrica. Rev.Bras.Enfermagem, Porto Alegre, v. 36, n.1, p. 2937, jan./mar. 1983.

PAIVA, M.M.S. et al. Avaliação das ações educativas em enfermagem para diabéticos: uma experiência de integração docente assistencial no hospital universitário - U.F.C.Fortaleza - CE. Rev.Bras.Enfermagem, Brasília, v. 39, n. 2/3, p. 55-65, abr./set. 1986.

PEREIRA, R.C.J. Implicações das ações educativas de enfermagem junto aos clientes. Rev. Gaúch.Enfermagem, Porto Alegre, v. 4, n. 1, p. 49-53, jan. 1983.

PIERIN, A.M.G. Orientação sistematizada do paciente hipertenso submetido a tratamento ambulatorial. Rev.Esc.Enfermagem USP, São Paulo, v. 20, n. 3, p. 193205, 1986.

- A pessoa com hipertensão arterial em tratamento no ambulatório: considerações gerais. Rev.Esc.Enfermagem USP, São Paulo, v. 22, n. 2, p. 223229, ago. 1988.

. A pessoa com hipertensão arterial em tratamento no ambulatório: estudo sobre os problemas, dificuldades e modificações decorrentes da doença e tratamento. Rev.Esc.Enfermagem USP, São Paulo, v. 22, n. 3, p. 273282, dez. 1988. 
A pessoa com hipertensão arterial em tratamento no ambulatório: influência de variáveis, conhecimento das complicações da doença e expectativa quanto à assistência de enfermagem. Rev.Esc.Enfermagem USP, São Paulo, v. 23, n. 1, p. 35-48, abr. 1989.

PIMENTA, C.A.M.; FUKUDA, C.L. Analgesia peridural domiciliar: a atuação da enfermeira. Rev.Bras.Enfermagem, Brasília, v. 47, n. 1, p. 31-35,jan./ mar. 1994.

POZZAN, R. et al. Experiência com um programa de educação para pacientes diabéticos com baixo nível sócioeconômico. Rev.Bras.Enfermagem, Brasília, v. 47, n. 3, p. 241-249, jul./set. 1994.

ROCHA, M.do P.S. Assistência de enfermagem em uma unidade de cuidados terciários ao diabético. Rev.Bras.Enfermagem, Brasília, v. 37, n. 3/4, p. 182-187, jul./dez. 1984.

SANTOS, B.R.L.; MENDES, E.E.M. Programa de assistência de enfermagem a clientes portadores de danos cardiovasculares, no ambulatório de um hospital de ensino de Porto Alegre-RS. Rev. Gaúch.Enfermagem, Porto Alegre, v. 4, n. 1, p. 61-73, jan. 1983.

SANTOS, B.R.L. Programa de assistência de enfermagem a cliente portadores de danos cardiovasculares no ambulatório de um hospital geral e de ensino de Porto Alegre, RS. Rev.Bras.Enfermagem, Porto Alegre, v. 36, n. 3/4, jul./dez. 1983.

SCAIN, S. F. Educação para a saúde a grupos de clientes diabéticos. Rev. Gaúch.Enfermagem, Porto Alegre, v. 7, n.2, p. 232-246, jul. 1986.

SCAIN, S.F.; OLIVEIRA, C.H.; FRANZEN, E. Aspectos de assistência de enfermagem ambulatorial no custo do tratamento de diabéticos. Rev. Gaúch.Enfermagem, v. 8, n. 2, p.149-167, jul. 1987.

SCOCHI, C.G.S. et al. O conhecimento de puérperas sobre o cuidado com o recém - nascido: avaliação da influência da internação em maternidade de um hospital - escola. Rev.Bras.Enfermagem, Brasília, v. 45, n. 1, p. 36-43, jan./ março 1992.

SERAFIM, D.; CAETANO, L.C.; BERNI, N.I.O. Atuação da enfermeira obstetra junto à gestante adolescente. Acta Paul.Enfermagem, São Paulo, v. 4, n. 1, p. 11-16, março 1991.

SILVA, M.E.K.; GONZAGA, F.R.S.R.; VERDI, M.M. Marco conceitual para a prática assistencial de enfermagem enquanto processo educativo em saúde. Rev.Bras.Enfermagem, Brasília, v. 45, n. 1, p. 54-59, jan./ março 1992.

\section{REFERÊNCIAS BIBLIOGRÁFICAS}

01. BARTLETT, E.E. Editorial: At last, a definition. Patient Educ.Couns. v. 7, p. 323-324, 1985.

02. BILLE, A.D. Practical approaches to patient teaching. Boston: Little-Brown, 1981.

03. DINES, A. What changes in health behavior might nurses logially expect from their health education work? Journal of Advanced Nursing, v. 20, p. 219-226, 1994.
SILVA PINTO, S.M.P. et al. Metodologia da assistência - uma nova estratégia de educação em saúde. Rev.Bras.Enfermagem, Porto Alegre, v. 36, n. 2, p. 177182, abr./maio/jun. 1983.

SIQUEIRA, M.M.; CASAGRANDE, L.D.R. Enfoque sistêmico aplicado à educação em saúde mental de adolescentes. Rev. Gaúch.Enfermagem, Porto Alegre, v. 6, n. 2, p. 175-185, jul. 1985.

TASHIRO, M.T.O.; SOUZA, M.F.; OLIVEIRA, S.D. Autocuidado no tratamento pelo método de Ilizarov - um estudo de caso. Rev.Bras.Enfermagem, Brasília, v. 48, n. 1, p. 46-50, jan. mar. 1995.

TEIXEIRA, M.E.M.; BARBOSA, M.A.; SILVA, L.F. Percepções dos pacientes quanto aos procedimentos invasivos no pós - operatório de cirurgias de grande porte. Rev.Bras.Enfermagem, Brasília, v. 47, n. 3, p. 250-257, jul./set. 1994.

TESSER, R.; CADORE, M. L. B. A importância da enfermagem na orientação e preparo ao paciente traqueostomizado. Rev. Gaúch.Enfermagem, Porto Alegre, v. 4, n. 2, p. 201-214, jul. 1983.

VALLE, E.G.; GUDES, M.V.C.; ALBUQUERQUE, M.T. Pósoperatório de cirurgia cardíaca: o que pensam e verbalizam os pacientes. Rev.Bras.Enfermagem, Brasília, v. 43, n. 1,2,3/4, p. 79-84, jan./dez. 1990.

VARGAS, G.O.P.; SCAIN, S.F. Educação alimentar e atividade física sistemática a clientes com excesso de peso e obesidade na consulta de enfermagem. Rev. Gaúch.Enfermagem, Porto Alegre, v. 3, n. 2, p. 165-174, jun. 1982.

VARGAS, G.O.P.; SCAIN, S.F. Educação para a saúde a grupo de obesos. Rev.Bras.Enfermagem, Porto Alegre, v. 36, n. 1, p. 38-49, jan./mar. 1983.

VOLPATTO, A. et al. Manual de orientação a pacientes portadores de diabetes mellitus. Rev. Gaúch.Enfermagem, Porto Alegre, v. 7, n. 2, p. 325-335, jul.1986.

WALDOW, V.R. Aprendizagem na velhice: aplicação das teorias de Rogers, Maslow e Brunner. Rev. Gaúch.Enfermagem, Porto Alegre, v. 8, n. 2, p. 244-260, jul. 1987.

ZAGO, M.M.F.; CASAGRANDE, L.D.R. As necessidades de aprendizagem dos pacientes laringectomizados. Rev. Gaúch.Enfermagem, Porto Alegre, v. 13, n. 2, p. 17-21, jul. 1992.

ZAGO, M.M.F. Considerações sobre o ensino do paciente cirúrgico. Rev.Esc.Enfermagem USP, v. 27, n. 1, p. 6771 , abr. 1993

04. GLANZ, K.; LEWIS, F.M.; RIMER, B.K. Health behavior and health education: theory, research and practice. San Francisco: Jossey-Bass Publishers, 1990.

05. PATTERSON, C.; TEALE, C. Influence of written information on patient's knowledge of their diagnosis. Age and Ageing, v. 26, n. 1, p. 41-42, jan/1997. 
06. RANKIN, S.H.; STAllingS, K.D. Patient education: issues, principles, practices. $3^{\text {rd }} \mathrm{ed}$. Philadelphia: Lippincott, 1996.

07. REDMAN, B.K. Patient education at 25 years: where we have been and where we are going. Journal of Advanced Nursing, v. 18, p. 725-730, 1993.

08. SALVADOR, A.D. Métodos e técnicas de pesquisa bibliográfica. 11. ed. Porto Alegre: Sulina, 1986.

09. STAMLER, L.L. Toward a framework for patient education: an analysis of enablement. Journal of Holistic Nursing, v. 14, n. 4, p. 332-347, 1996.
10. TAYLOR, V. Health education: a theoretical mapping. Health Education Journal,v. 49, n. 1, p. 13-14, 1990.

11. TONES, K.; TILFORD, S; ROBINSON, I. Health education: effectiveness and efficiency. $2^{\text {nd }} \mathrm{ed}$. London: Chapman \& Hall, 1991.

12. ZAGO, M.M.F. O ritual de orientação de pacientes pelos enfermeiros cirúrgicos: um estudo etnográfico. 1994. 154 p. Tese (Doutorado) Escola de Enfermagem de Ribeirão Preto, Universidade de São Paulo. 